\title{
DESIGUALDAD Y FRONTERA NORTE MEXICANA: UNA APROXIMACIÓN DESDE LOS OBJETIVOS DE DESARROLLO SOSTENIBLE
}

\author{
INEQUALITY AND THE NORTHERN MEXICAN BORDER: A SUSTAINABLE \\ DEVELOPMENT APPROACH
}

\author{
Manuel Antonio Jiménez Castillo ${ }^{1}$
}

\begin{abstract}
Resumen
Este trabajo pretende arrojar luz sobre cómo la extrema desigualdad que aflige a la frontera norte mexicana condiciona el cumplimiento de los objetivos del desarrollo sostenible. A través de los conceptos de "resiliencia" y de "asimetría de poder" se examinará cómo la desigualdad actúa como un pernicioso actor político descompensando el sentido originario de la acción política. Para tal fin, se toman cinco de los objetivos de desarrollo sostenible (ODS) con mayor incidencia sobre la desigualdad con el fin de conocer el estado real de su cumplimiento. Igualmente, se conceptualizarán sus efectos potenciales a la hora de dar logro a tales fines. Se concluirá aseverando sobre la existencia de un efecto perverso de la desigualdad sobre aquellas políticas dadas a convertir los logros del milenio en un asunto sostenible.
\end{abstract}

Palabras Claves: Capacidades; Desarrollo Humano; Desigualdad; Sostenibilidad

\section{Abstract}

This paper is an attempt to shed light on how the extreme inequality affecting the northern Mexican border conditions the fulfillment of

doi: http://dx.doi.org/10.15359/eys.21-50.1

Fecha de recepción: 31-05-16. Fecha de reenvío: 09-09-16/ 14-09-16. Fecha de aceptación: 26-09-16. Fecha de publicación: 26-09-16

${ }^{1}$ Doctor en Ciencias Económicas. Investigador y profesor, El Colegio de la Frontera Norte, Sede Nuevo Laredo, México. Correo electrónico: antoniojcastillo@colef.mx

Manuel Antonio Jiménez Castillo

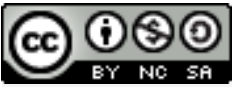

Revista Economía y Sociedad by Universidad Nacional is licensed under a CreativeCommons Reconocimiento-NoComercial- 
sustainable development goals. Using the concepts of "resilience" and "power asymmetry", the author analyzes how inequality, like a pernicious political actor, destabilizes the original sense of the political action. To this end, the five Sustainable Development Goals (SDG) that are most related to inequality are analyzed to verify if they are actually being met. In addition, the potential effects they will have when met will be conceptualized. We conclude by restating the existence of a perverse effect of inequality on those policies established to achieve sustainable development goals.

Keywords: Capabilities; Human Development; Inequality; Sustainability

\section{Introducción}

Con la celebración de la Conferencia de Desarrollo Sostenible Río+20 a finales de 2012 se reconocía a nivel global el desigual resultado alcanzado en el cumplimiento de unos objetivos del milenio (ODM) que Ilegaban a su fin. Una etapa que coincidía con el establecimiento de un nuevo grupo de trabajo encomendado en facilitar el diseño de un conjunto de propuestas originales con las cuales se fijan las premisas para una nueva agenda mundial "Agenda 2030". De las conclusiones de esta etapa resultaba un acuerdo que se cerraba tres años después con la llamada Cumbre Mundial de Desarrollo Sostenible. En esta se acordaba una serie de objetivos de desarrollo sostenible (ODS) que fijaría los principios del discurso del desarrollo para la próxima década y media. Aunque son muchos los matices que diferencian ambas agendas (Nayyar, 2011), los ODS nacían desde una narrativa bipolar donde se incorporaban los conceptos de desigualdad y sostenibilidad, los cuales fueron desatendidos en la agenda anterior (Fukuda-Parr, et al.,2014). Esta nueva filosofía respondía, entre otras, a la denuncia del Programa de Naciones Unidas al Desarrollo (PNUD) ante el incesante incremento de la desigualdad en el mundo (Vandemoortele, 2014) desde una visión que incluyera "no solo una cuestión de ampliación de opciones (...) sino de cuán seguros son estos logros". Una seguridad que exigía como condición sine qua non la reducción de la desigualdad en todas sus dimensiones haciéndola a su vez extensible a "todos los grupos de ingresos y sociales relevantes" (High Level Panel, 2013, p.19). El protagonismo creciente de la desigualdad en la nueva agenda como garante de una demandada "seguridad en el logro" apunta, sin embargo, a un giro copernicano, pues le exige transitar como predicado de las distintas reacciones mercantiles(propias de la escuela neoclásica) como fundamento del bienestar humano. Desde este sentido, la desigualdad se configura como variable focal de la nueva agenda, donde el sentido de resiliencia (libertad negativa), la cual fundamenta el marco de los nuevos objetivos, ofrece a aquella una serie de atributos potencialmente (activadores).

Asimismo, unos atributos que para la nueva agenda la articulan como "actor político" al presentarse como sujeto de la acción (económica). Su tratamiento multidimensional le permite

2

Manuel Antonio Jiménez Castillo

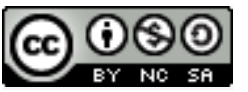

Revista Economía y Sociedad by Universidad Nacionalislicensedunder a CreativeCommons Reconocimiento-NoComercial- 
adaptarse como elemento capaz de afectar el sentido prioritario de las políticas públicas, así como su resultado. Desde esta perspectiva, lo desigual no se agotaría desde un leimotiv relacional y resultante de una actividad entre agentes, sino como fundamento mismo de su realidad posible. Esta perspectiva auto causal, la hace especialmente atractiva como objeto para entender de qué manera se conjuga, a partir de sus efectos, los distintos escenarios empíricos posibles de desarrollo. Específicamente, en este trabajo gana consistencia el estudio de la desigualdad, así como sus efectos sobre los ODS en la frontera norte mexicana (FNM). EI elevado grado de desigualdad, ya sea en términos de indicadores de ingresos como el de Gini (49\%) o de su carácter más social (Coneval, 2014) lo hacen particularmente apropiado para corroborar hasta qué punto la desigualdad fronteriza actúa no solo como protagonista normativo de la agenda, sino como actor "operativo" para el cumplimiento de los ODS.

La hipótesis de este trabajo pretende no solo introducir en origen y relevancia la desigualdad en la nueva agenda sostenible, sino que, a partir de ello, corroborar como sus efectos a nivel operativo son particularmente evidentes para la FNM. Esto permitiría ilustrar especulativamente de qué modo elementos que comúnmente son dados a mitigar la desigualdad consiguen, en un excepcional efecto perverso, incrementarla. En tales circunstancias, la desigualdad, sin liberarse de su carácter relacional, pero sin disolverse en él esta, se presentaría como fenómeno causal que en su devenir subvierte el cumplimiento de los ODS. Desde este sentido, la corroboración de la hipótesis pasa por el cumplimiento de los siguientes objetivos; a) examinar la relevancia conceptual de la desigualdad en la nueva agenda del desarrollo 2030 desde el marco de las teorías de las capacidades, b) en un ejercicio de prospectiva, donde se ilustra cuantitativamente cómo se sitúa la frontera norte en el cumplimiento de aquellos ODS más afectados por la desigualdad, c) finalmente, y aprovechando la focalidad de la desigualdad para la nueva agenda, analizaremos cómo para la FNM esta adquiere unas características propias de "espiral viciosa" que problematizan la eficacia de cualquier política dada al cumplimiento de tales objetivos.

Con el fin de cumplir con tales objetivos es necesario no solo identificar el tercero de estos, pues, aunque sea el elemento quizás más innovador del trabajo no podría entenderse sino es vinculado al marco histórico-teórico y empírico (objetivos 1 y 2) que lo vertebran y dan sentido. Por lo tanto, el plan de trabajo se presenta del siguiente modo: en un primer apartado se introducirán las implicaciones del nuevo rol de la desigualdad en el discurso de los ODS, en donde se toma como referente el concepto de "resiliencia" trabajado por el PNUD. Por medio de este concepto examinaremos las consecuencias analíticas de lo que supone un perfeccionamiento teórico del enfoque de Amartya Sen y por ende de los objetivos de desarrollo del milenio (ODM) desde la inclusión de lo que de procesos atesora la libertad real en el forjamiento de las capacidades.

En segundo lugar, de su relevancia en la nueva agenda del desarrollo, se ilustrará de forma descriptiva el estado actual para la frontera desde aquellos ODS más vinculados con la equidad.

Manuel Antonio Jiménez Castillo

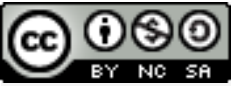

Revista Economía y Sociedad by Universidad Nacional is licensed under a CreativeCommons Reconocimiento-NoComercial- 
Con ello pretendemos dar perspectiva empírica al esfuerzo que requeriría la FNM para hacer cumplir tales objetivos.

En un tercer momento se expondrá analíticamente cómo la desigualdad en la FNM se presenta como condicionante causal para con el cumplimiento de los objetivos de sostenibilidad. Desde los conceptos de "espiral viciosa" y "asimetría de poder" nos aproximaremos a un concepto de desigualdad "fronteriza" donde su naturaleza transmuta a causa de sí misma y afecta la naturaleza de aquellos instrumentos de política económica y social diseñados para mitigarla; para tal objeto, se analizará cómo la desigualdad se desenvuelve en distintos escenarios de política económica y social. En un último apartado se concluirá con los aspectos más destacados del texto.

\section{El concepto de desigualdad en el discurso de los ODS}

Notable ha sido el revuelo generado acerca de las implicaciones tanto exógenas (Martínez, 2013) como endógenas (Fukuda-Parr, 2010; Nayyar, 2011; Vandemoortele, 2014) del tránsito a la nueva agenda sostenible. Para lo cual existe un robusto consenso académico de que aquello que propició Rio+20 fue una conjunción de sucesos materializados en una serie de debilidades metodológicas propias de unos objetivos del milenio incapacitados para integrar los nuevos acontecimientos internacionales emergentes (Fukuda-Parr, 2010). No obstante, parece ser que el contenido de los acuerdos resultantes con el quehacer frente a tales debilidades no ha solventado todos los problemas asociados a aquellas. El hecho de que estos nuevos objetivos se vertebren desde el sentido más prosaico de los estudios del desarrollo (cooperación para) ha perjudicado en exceso tanto a su realismo como a la capacidad de influencia y legitimación de las políticas globales de desarrollo (Fukuda-Parr, 2010).

Por consiguiente, introducirse en los fundamentos teóricos del desarrollo adaptado por Naciones Unidas implica enfrentarse con el enfoque de las capacidades. Desde su primer informe se aboga por entender el desarrollo como el resultado de una expansión de las oportunidades y elecciones reales de los individuos (Sen, 1999), frente a aquella idea esencialista del crecimiento económico per se que había monopolizado hasta entonces las teorías contemporáneas (PNUD, 1990). Desde la variable focal de libertad como capacidad, Amartya Sen conseguía construir un enfoque de justicia social donde el punto de inflexión fuera la calidad de vida (bienestar+ agencia) desde aquello que el sujeto es capaz de lograr realmente (Sen, 2009). Logro y la libertad en el logro (Sen, 1999) se presentaban conjuntamente como fundamento del bienestar real de los individuos. Su incorporación a la nueva agenda sostenible (PNUD, 2014) permite articular la educación, sanidad, empleo, participación social, etcétera, ya no como necesidades por satisfacer (Max-Neef, 1986) y sí como fines potencialmente capacitadores (PNUD, 1999; Sen, 2009).

4

Manuel Antonio Jiménez Castillo

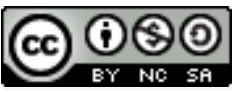

Revista Economía y Sociedad by universidad Nacionalislicensedunder a CreativeCommons Reconocimiento-NoComercial- 
A pesar de que la teoría seniana inspira desde sus fundamentos los ODM, aquella lo hacía restringida al logro (libertad de oportunidad) a partir de la materialización de una serie de objetivos cuantificables. Con la aprobación de la nueva agenda de desarrollo sostenible se alcanza a recuperar lo que de la libertad real quedaba desplazado en Sen (2009) al poner el acento sobre la seguridad en el logro, y, por consiguiente, sobre la libertad de procesos (Jiménez-Castillo, 2016). La cuestión ya no versa sobre el cumplimiento de los logros en sí mismos y sí sobre la posibilidad de hacer sostenible su consecución. Este aspecto abre la puerta a varias innovaciones con implicaciones analíticas y operativas para la nueva agenda sostenible. Por un lado, se realiza un acercamiento al concepto de libertades reales desde las oportunidades para vivir una vida deseada (Sen, 1999), pues se incorpora el concepto de vulnerabilidad (tan ausente en las capacidades individuales) en la arquitectura de los nuevos objetivos (PNUD, 2014).

Seguidamente, y extraíble del punto anterior, la incorporación de la seguridad en el logro integra aquellos elementos que, ajenos a la voluntad individual, la determinan en la posibilidad para controlar y establecer las propias acciones deseables (Deneulin, 2011). Este segundo aspecto es tratado por el PNUD por medio del concepto de resiliencia humana y viene a completar el vacío analítico (lo social de la libertad) en Amartya Sen (Deneulin, 2006; 2011; Jiménez-Castillo, 2016). Un vacío que se presenta en la propia interpretación del concepto de libertad pues la delimita a un estado de oportunidad, pues aparta todas aquellas condiciones materiales y estados resultantes del entorno social que determinan el surgimiento de las oportunidades reales (Cejudo, 2007). Esta circunstancia, de la que su autor es plenamente consciente, le impide capturar aquellas interferencias, que, derivadas de las restricciones sociales e institucionales, entre otras, forman parte del proceso de empoderamiento. Este hecho que condiciona todo el éxito operativo y el realismo normativo del enfoque (Deneulin, 2011; Nussbaum, 2012) es corregido en la nueva agenda sostenible al incorporar aquellos elementos del entorno social (libertad de procesos) para cada uno de sus objetivos (FukudaParr, 2012).

Desde este perfeccionamiento analítico la desigualdad adquiere un cariz ajeno al heredado de la agenda del milenio. Para los ODM el verdadero progreso del desarrollo humano residía en una ampliación de las opciones, y por tanto, la desigualdad quedaba resignada a una distribución no-equitativa de los logros alcanzables, solo en uno de los ocho objetivos se hace referencia a la disparidad (Fukuda-Parr, 2010). En cambio para los ODS lo relevante subyace en la idea de "cuán seguros son estos logros (...)" (Pnud, 2014, p.8). Lo focal del asunto ya no se encuentra en la idea del logro en sí, sino en su sostenibilidad.

Al poner en valor lo "desigual de la seguridad" gana sentido hablar en términos de lo sostenible, y por tanto, de aquellos estados que facilitan resultados propicios. El concepto de desigualdad se reactualiza desde un principio de discusión más elevado -la seguridad frente al logro-apuntando con ello a unos atributos del todo activadores (desigualdad como "actor 
político"). Más allá de tal relevante acontecimiento, que por otro lado ilustrará lo que de particular se le atribuirá a la desigualdad en la frontera, en nada lo libera de la espinosa crítica maximalista, aun cuando los logros del milenio respondían a principios empíricos mediados normativamente. Por ejemplo, incluso alcanzando el ODM 1 tal y como sostiene Naciones Unidas, parecería incoherente celebrar una transposición de la desigualdad hacia la seguridad cuando más de 700 millones de personas viven aún con menos de 1,25 dólares diarios.

A pesar del precio maximalista que demanda un "mayor entendimiento" (seguridad frente al logro), los ODS sí que rompen con el sentido de "universalidad abstracta" en el que quedaba restringida la distribución en la agenda del milenio ${ }^{2}$. El carácter interrelacionado de la nueva agenda (Le Blanc, 2015), su alcance a nuevas dimensiones del desarrollo (de 8 a 17 objetivos), su intrínseca relevancia como objetivo, su ponderación sobre múltiples aspectos de la pobreza, y su carácter universal hacen de la desigualdad un elemento capaz de recoger fielmente aquellas dinámicas que "inhabilitan la habilidad de las personas para actuar". A pesar de que la distribución gana ahora toda su significación no se ultima con ello la operatividad del enfoque de las capacidades. De tal manera que con una plena adaptación de la distribución en los nuevos objetivos quedará para sí operativo el enfoque humano de capacidad. La libertad en el logro no agota la sostenibilidad del logro que requiere para sí un ejercicio de la voluntad del agente que en última instancia escapará al contenido de la agenda.

\section{La desigualdad en la nueva agenda sostenible para la FNM}

Una vez señalada la relevancia de la desigualdad en el marco de la agenda sostenible el siguiente paso exige describir empíricamente el estado de la cuestión para la FNM. Solo por medio de un análisis descriptivo se puede alcanzar una idea clara de cuán lejos se encuentra la frontera de garantizar su cumplimiento. Para ello, se tomarán hasta cinco indicadores de desarrollo sostenible donde se procura una mayor ponderación distributiva. Si bien, en este ejercicio se encontrarán una serie de limitaciones. En primer lugar, la reciente instauración de los nuevos objetivos impide que existan series estadísticas que reflejen fielmente cada uno de los objetivos aprobados. Por otro lado, el deficiente material estadístico en la FNM hace que muchas de las mediciones sobre indicadores de desarrollo humano se encuentren sesgadas y desactualizadas (Scott, 2010). Para combatir la primera de las limitaciones se tomará aquella serie de indicadores del milenio que, para el dato más reciente, se aproximen descriptivamente a los ODS elegidos. Aunque ello supone un sesgo notable, que en nada facilita la tarea encomendada, pero sí permite ilustrar con perspectiva el basamento desde el que la frontera afronta el nuevo reto. Con referencia al segundo de los límites se utilizarán las estadísticas del

\footnotetext{
${ }^{2}$ La desigualdad es reconocida a partir de principios más elevados como la reducción de la pobreza extrema, la mortalidad infantil, combatir el VIH/SIDA, el paludismo y otras enfermedades, garantizar la sostenibilidad del medioambiente o fomentar una asociación mundial por el desarrollo.
}

6

Manuel Antonio Jiménez Castillo

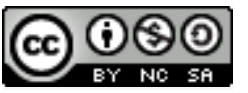

Revista Economía y Sociedad by Universidad Nacionalislicensedunder a CreativeCommons Reconocimiento-NoComercial- 
PNUD sobre los ODM para cada región de la FNM. Los resultados de cada indicador serán clasificados atendiendo a la metodología de las "tres erres". Asimismo, se vislumbrará un estado de Reforma para aquellos objetivos que se muestren altamente logrables, pero de los que se requiere continuidad. En un estado de Revolución se clasificarán aquellos para los que el cumplimiento del objetivo es poco probable y del que se requeriría una mayor contribución para alcanzarlo. Finalmente, con Reversión se muestran aquellos objetivos cuyo alcance depende de revertir todas las acciones políticas implementadas hasta el momento.

Tabla 1.

Porcentaje de mujeres en el total de asalariados en el sector no agropecuario (ODM3.2)

\begin{tabular}{lccl}
\hline $\begin{array}{c}\text { Estados Fronterizos } \\
\text { Norte y País }\end{array}$ & $\begin{array}{c}\text { Línea Base } \\
(1995)\end{array}$ & $\begin{array}{c}\text { Dato más reciente } \\
(2014)\end{array}$ & $\begin{array}{l}\text { Prospectiva } \\
\text { Meta ODS5 }\end{array}$ \\
\hline Baja California & ND & 40.9 & Revolución \\
Chihuahua & ND & 39.6 & Revolución \\
Coahuila & ND & 37.8 & Revolución \\
Nuevo León & ND & 36.5 & Revolución \\
Sonora & ND & 43.3 & Revolución \\
Tamaulipas & ND & 41.6 & Revolución \\
México & 38.1 & 41.4 & Revolución \\
\hline
\end{tabular}

Fuente: elaboración propia desde PNUD (2014)

El primero de los ODS 5.5 elegido tiene que ver con la "participación plena y efectiva de las mujeres y la igualdad de oportunidades a todos los niveles (...)". Para su medición se tomará el ODM 3.2 correspondiente. Aunque este indicador es mucho más restrictivo al limitar la igualdad al campo de la empleabilidad laboral no agrícola, atiende a la equidad de género desde la posibilidad del sexo femenino de empoderarse a partir de la ruptura con su rol social tradicional. Como se desprende del Tabla $\underline{1}$, solo poseemos datos comparativos a nivel nacional los cuales revelan un incremento general de 3.3 puntos porcentuales. Con respecto a las entidades federativas se observa que solo Sonora y Tamaulipas superan la media nacional quedando Nuevo León a casi $7 \%$ de la primera. Ninguna de las entidades alcanza la media de la región Latinoamérica y Caribe que se sitúa en el $45 \%$ y tan solo dos de las seis supera la media mundial del $41 \%$. En atención a los datos, un escenario de Revolución se antoja razonable para cumplir con el ODS 5. 
Tabla 2.

Porcentaje de la población con acceso sostenible a fuentes mejoradas de abastecimiento de agua (ODM7.8)

\begin{tabular}{lccc}
\hline $\begin{array}{c}\text { Estados Fronterizos } \\
\text { Norte y País }\end{array}$ & $\begin{array}{c}\text { Línea Base } \\
(1995)\end{array}$ & $\begin{array}{c}\text { Dato más reciente } \\
(2014)\end{array}$ & $\begin{array}{c}\text { Prospectiva } \\
\text { Meta ODS6 }\end{array}$ \\
\hline Baja California & 79.9 & 95.9 & Reforma \\
Chihuahua & 87.6 & 94.6 & Reforma \\
Coahuila & 91.9 & 98.3 & Reforma \\
Nuevo León & 92.9 & 96.6 & Reforma \\
Sonora & 91.0 & 96.6 & Reforma \\
Tamaulipas & 80.9 & 95.9 & Reforma \\
México & 78.4 & 90.9 & Reforma \\
\hline
\end{tabular}

Fuente: elaboración propia desde PNUD (2014)

Tabla 3.

Porcentaje de la población con acceso a servicios de saneamiento mejorados (ODM7.9)

\begin{tabular}{|l|c|c|c|}
\hline $\begin{array}{c}\text { Estados Fronterizos } \\
\text { Norte y País }\end{array}$ & $\begin{array}{c}\text { Línea Base } \\
(1990)\end{array}$ & $\begin{array}{c}\text { Dato más reciente } \\
(2010)\end{array}$ & $\begin{array}{c}\text { Prospectiva } \\
\text { Meta ODS7 }\end{array}$ \\
\hline Baja California & 64.3 & 92.6 & Reforma \\
\hline Chihuahua & 64.4 & 91.8 & Reforma \\
\hline Coahuila & 66.6 & 95.3 & Reforma \\
\hline Nuevo León & 92.9 & 95.1 & Reforma \\
\hline Sonora & 63.3 & 89.0 & Reforma \\
\hline Tamaulipas & 56.5 & 86.7 & Reforma \\
\hline México & 58.6 & 87.7 & Reforma \\
\hline
\end{tabular}

Fuente: elaboración propia desde PNUD (2014)

Para los ODS 6.2-7.5 relativo al acceso equitativo de servicios de saneamiento y mejora tecnológica para servicios de energía modernos se han elegido los indicadores ODM 7.8 y 7.9 respectivamente. En relación al primero de estos, la posibilidad para lograr en 2030 un acceso equitativo a servicios de saneamiento e higiene adecuados para todos (ODS 6.2) se antoja muy favorable a tenor de la información que se desprende del Tabla $\underline{2}$. Se ha experimentado un incremento medio de alrededor del 9\% para las entidades de federativas en los últimos 20 años. Todas estas se encuentran por encima de la media mundial situada en el $91 \%$ y solo con la excepción de Chihuahua superan el promedio para la región de América Latina y Caribe (95\%). Un perfil aún más optimista se desprende en relación con la Meta ODS7 que coincide con el indicador del Milenio 7.9.

8 
De la Tabla $\underline{3}$ se revela como el acceso a servicios de saneamiento mejorado ocupa casi todo el espectro poblacional de las entidades con un incremento del $40 \%$ de media frente al año base. Esto supone un mejoramiento muy significativo no solo frente a la media de la Región Latina que queda fijada en un $83 \%$, sino que para entidades como Baja California o Nuevo León supera en más de 20 puntos porcentuales la media mundial (68\%). Un escenario de Reforma parece ser la estrategia más adecuada para asegurar el cumplimiento de ambos objetivos.

Tabla 4.

Tasa de mortalidad en niños menores de 5 años

\begin{tabular}{lccl}
\hline $\begin{array}{c}\text { Estados Fronterizos } \\
\text { Norte y País }\end{array}$ & $\begin{array}{c}\text { Línea Base } \\
(1990)\end{array}$ & $\begin{array}{c}\text { Dato más reciente } \\
(2013)\end{array}$ & $\begin{array}{l}\text { Prospectiva } \\
\text { Meta ODS3 }\end{array}$ \\
\hline Baja California & 43.4 & 17.6 & Reforma \\
Chihuahua & 46.1 & 18.7 & Reforma \\
Coahuila & 33.6 & 13.7 & Reforma \\
Nuevo León & 27.5 & 11.4 & Reforma \\
Sonora & 35.6 & 13.0 & Reforma \\
Tamaulipas & 34.0 & 15.3 & Reforma \\
México & 41.0 & 15.8 & Reforma \\
\hline
\end{tabular}

Fuente: elaboración propia desde PNUD (2014)

En referencia al ODS 3.2 se ha tomado como indicador el ODM 4.1 por su similitud significativa. De la Tabla $\underline{4}$ se extrae como FNM se ajusta a la tendencia media mundial en la que la reducción de la mortalidad infantil ha sido generalizada y notable pasando de 90 a 43 muertes por cada 1.000 niños. Si bien, los datos para la FNM son prometedores pues consigue reducir en más de un $14.2 \%$ de media la mortalidad en cada una de las regiones, y obtiene resultados parejos a los países desarrollados (15) y muy por debajo de la media mundial (43). A nivel de bloques regionales la gran mayoría de los estados se encuentran por debajo de la media latinoamericana y caribeña (17) con la excepción de Baja California y Chihuahua. En cuestión de mínimos "al menos hasta 25 por cada 1.000 nacidos" la FNM ya habría cumplido con el ODS 3.2, para lo cual aplazaría la cuestión de máximos "para 2030 poner fin a las muertes evitables de niños menores de 5" a la capacidad de dar continuidad (Reforma) a los logros alcanzados. 
Tabla 5.

Relación entre ocupación y población en edad de trabajar

\begin{tabular}{lccl}
\hline $\begin{array}{c}\text { Estados Fronterizos } \\
\text { Norte y País }\end{array}$ & $\begin{array}{c}\text { Línea Base } \\
(1995)\end{array}$ & $\begin{array}{c}\text { Dato más reciente } \\
(2014)\end{array}$ & $\begin{array}{c}\text { Prospectiva } \\
\text { Meta ODS8 }\end{array}$ \\
\hline Baja California & ND & 58.4 & Reversión \\
Chihuahua & ND & 56.2 & Reversión \\
Coahuila & ND & 58.2 & Reversión \\
Nuevo León & ND & 57.9 & Reversión \\
Sonora & ND & 60.5 & Reversión \\
Tamaulipas & ND & 57.9 & Reversión \\
México & 55.3 & 56.9 & Reversión \\
\hline
\end{tabular}

Fuente: elaboración propia desde PNUD (2014)

La última de las metas que atenderemos es la referida al pleno empleo. Para ello se utilizará la meta ODS 8.5 desde el indicador del milenio ODM 1.5. Desde un primer momento se desprende estadísticamente lo abultado de los problemas de empleabilidad en toda la frontera. Al consultar los datos específicos por entidades, se observa como el mejor de los registros es el correspondiente a Sonora que mantiene aun así el 39.5\% de personas desempleadas o expulsadas del mercado formal. Este hecho que acompaña a toda la economía mexicana en general supone quizás una de las metas más arduas que deberá de afrontar FNM. Además, su cumplimiento está sometido a cambios estructurales de la economía y la sociedad mexicana que trascienden la implementación de políticas de alcance meramente coyuntural. Un estado de Reversión pareciera ser el más adecuado a tenor del enorme esfuerzo de recomposición que le toca a una economía cuya estructura socioeconómica dual y concentrada da visos de plausible incumplimiento.

\section{La desigualdad ante su espejo}

La cuestión que sobreviene al análisis descriptivo de los ODS comparte relación con el conjunto de medidas y acciones encaminadas a dar garantías de su cumplimiento. En este sentido, los aspectos que rondan este asunto se pueden sintetizar en la siguiente pregunta: ¿se encuentra la FNM en condiciones, y si fuera así en qué medida, de garantizar el cumplimiento de la agenda sostenible en los plazos acordados? La respuesta a esta y otras preguntas podría apuntar a muy diferentes hipótesis. Empero en este apartado se ilustrará el mecanismo por el cual la desigualdad puede, más allá de manifestarse como resultado de reacciones descompensadas entre oferentes y demandantes (Dungey \& Jacobs, 2010), actuar como un verdadero "actor político", y como tal, atribuirse para sí, influencia sobre el cumplimiento de los objetivos. Lo que proponemos a fin de cuentas es que a elevadas tasas de desigualdad y pobreza (Coneval, 2014), esta se convierte responde a sí misma, de modo que al trascender su actitud relacional llegue a constituirse como responsable de tales resultados. En un sentido muy particular, la desigualdad 10

Manuel Antonio Jiménez Castillo

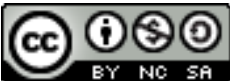

Revista Economía y Sociedad by Universidad Nacionalislicensedunder a CreativeCommons Reconocimiento-NoComercial- 
reactualiza las motivaciones económicas canalizándolas hacia un estado que refuerza una estructura permanentemente asimétrica entre los distintos agentes económicos. No se agota como resultado que apela a principios normativos de corrección extra-mercantiles (intervención estatal), sino que en su autonomía determina la propia naturaleza de tales intervenciones.

A partir de esta resignificación operativa (con un fuerte impacto normativo)de la desigualdad se podría explicar no solo las limitaciones que encuentra la frontera para implementar los objetivos derivados de la agenda sostenible, sino también el modo en que las políticas orientadas a tal fin se ven específicamente afectadas (Coneval, 2014). La desigualdad actúa precisamente predeterminando el sentido de los instrumentos destinados a combatirla, en donde desplaza de la ecuación aquel conjunto de causas (asimetrías del mercado, ineficiencia en el diseño de las políticas públicas, mal-intencionalidad, etcétera) que tradicionalmente explican su razón de ser. No es que la desigualdad ocurra por razón de aquellas, sino que más bien son aquellas las que inmersa bajo un clima de asimetría radical se ven determinadas por esta. Este efecto se asemeja a la lógica que gobierna el concepto de "asimetría de poder" en Weber según el cual; ante una mayor asimetría en la relación entre dos elementos dados $A$ (acción) y B (reacción), mayor será la descompensación entre la naturaleza de la acción A (instrumentos dados a combatirla) y su resultado B (reducción de la desigualdad). Un claro ejemplo lo muestra la política de gasto público orientada a reducir el impacto de la desigualdad en México. En su conjunto el gasto total (acción) es regresivo; el 10\% más pobre de la población recibe $7.8 \%$ de esos recursos, mientras el $10 \%$ más rico recibe el $16.7 \%$. El $60 \%$ de todo el gasto se concentra en los grupos de mayores ingresos (reacción desigual) (Scott, 2011). De este caso particular, entre muchos otros, se induce como la propia acción encaminada a refrenar la desigualdad se conforma desde una asignación asimétrica exante que refuerza su propia espiral.

Probablemente, la dificultad para identificar el punto " $x$ " donde la dispersión entre dos elementos generará un estado de "asimetría de poder" hace que esta hipótesis no haya sido respaldada académicamente con la relevancia que merece.

Ahora bien, al ser conscientes de esta circunstancia y de la dificultad que supone para la intención de este trabajo examinar la desigualdad como un instrumento de política económica surge la siguiente pregunta, ¿̇en qué grado se puede identificar una situación de "asimetría de poder" donde la desigualdad queda vinculada como un "actor político"? Al desviarse del sentido relacional de la desigualdad se muestra una ruptura en términos lógicos, pues no parece razonable que aquello dado como un "estado entre dos términos" pueda favorecer un sistema de determinación lineal causa-efecto. Sin embargo, más allá de las críticas resultantes, no se vislumbran otras alternativas (más que la especulativa) que de algún otro modo pudieran explicar de manera sistemática como las distintas políticas económicas y sociales en la FNM tienden a incrementar la desigualdad que estaba destinada a ser reducida. O en otras palabras, si la desigualdad no fuera causa de sí misma, no podría entenderse coherentemente los efectos perversos de una política macroeconómica de enfoque financierista cuyos efectos sobre la

Manuel Antonio Jiménez Castillo

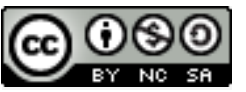

Revista Economía y Sociedad by Universidad Nacional is licensed under a CreativeCommons Reconocimiento-NoComercial- 
política fiscal auguran ineficiencia (captura de gasto público por parte de grupos de interés) y desigualdad (escasos recursos y capacidad redistributiva), así como regresividad y asimetría con respecto a la política social (Coneval, 2014).

Al atender al primero de estos, y subrayando que el marco financiarista se ha instalado en países con una desigualdad ajena a las propiedades atribuidas en esta hipótesis, sí vemos que sus efectos parecen ser determinantes para aquella, pero no en lo concerniente a las debilidades intrínsecas de un enfoque incapacitado para generar políticas expansivas que fomenten el desarrollo; "policy cannot be reduced to a means of reducing government deficits (...) it is a powerfulinstrument in thequestfor full employment and economic growth" (Nayyar, 2011, 344), sino preferentemente a su impacto sobre una arquitectura de bienestar social muy restringida a la hora de esquivar los efectos de la política monetaria ortodoxa (Esquivel, 2010). Tales restricciones afloran desde una estructura fiscal ineficiente y asimétrica. La capacidad de la política fiscal, para compensar los desajustes del mercado, se encuentra muy restringida, pues a la escasa capacidad para generar recursos propios se le une su limitada capacidad redistributiva.

Con un nivel de desigualdad entre actores económicos tan elevado, la presión fiscal del país es la más baja de la OCDE 18.8\% (Cepal, 2010). Este hecho se explica, entre otras, por una alta tasa de informalidad que hace muy costoso y limitada la recaudación en amplios sectores económicos (Johnson, et al., 1998) una estructura fiscal que favorece a las rentas más altas ( $7.3 \%$ de presión fiscal frente al 50\% de la OCDE), altas exenciones a las rentas del capital, importante dependencia de los impuestos a hidrocarburos (Scott, 2011), así como la existencia de grupos "extractores de rentas" (Rosignolo \& Gómez 2011). Con respecto a la capacidad redistributiva, la elevada desigualdad prefiscal fomenta una estructura donde se intensifica la presión impositiva indirecta e incrementa la pobreza, así como las barreras y costes para hacer accesible la provisión de bienes públicos (Scott, 2011). Ello acontece por problemas en el diseño mismo de los programas sometidos a "factores históricos e inerciales que responden a diseños institucionales y compromisos muy adversos al cambio" así como a la existencia de subsidios al consumo donde su regresividad ha beneficiado principalmente a los grupos de ingresos medios y altos.

Otro aspecto que ilustra el modo en el que la desigualdad actúa como actor político lo encontramos en las propias de bienestar social. La reacción de las políticas de gasto público en la FNM se puede explicar como causa del distanciamiento "gap" entre el policy-maker y la población objetivo. Su dilatada dispersión, provocada por una desigualdad de fondo irrenunciable, albergaría el contenido mismo de aquellas reacciones generadas por una falta de "simpatía" (conexión con). Esta ausencia simpática condiciona las políticas "públicas" dadas a remediar y manifestadas en una doble dimensión; por un lado, lo hace de manera restrictiva, puesto que limita el acceso a tales recursos por parte de la población más pobre. Solo el $29.6 \%$ de la población de la frontera tiene acceso general a la seguridad social mientras que hasta el 12

Manuel Antonio Jiménez Castillo

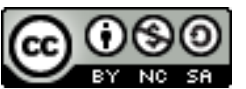

Revista Economía y Sociedad by Universidad Nacionalislicensedunder a CreativeCommons Reconocimiento-NoComercial- 
$36.8 \%$ de las transferencias de los programas de superación de la pobreza llega solo al primer quintil (Coneval, 2014). Esta limitación se hace efectiva a partir de los (des)incentivos y desconocimiento asociados a un segmento de la población excluido de los canales formales (Cortés \& De Oliveira, 2010). A ello se añaden factores inerciales e ideológicos (propios de tal distanciamiento entre actores) que acaban favoreciendo la propagación de grupos de interés organizados (Coneval, 2014). Tales factores terminan condicionando el buen diagnóstico y seguimiento de las políticas públicas que frecuentemente restringe el acceso gratuito a la población más vulnerable (Scott, 2010).

Por otro lado, termina siendo regresiva. Una regresividad que se muestra tanto en la asignación del gasto como en su resultado. Con respecto al primero de ellos, el $10 \%$ más pobre de la población recibe el $7.8 \%$ del gasto dirigido a combatir la desigualdad, mientras que el $10 \%$ más rico recibe el $16.7 \%$. De acuerdo a los datos de Scott $(\underline{2010}, 60)$ el sistema de seguridad social representa el rubro más regresivo de todo el gasto social, "mientras que el decil más rico obtiene una cobertura del 90\%; el decil más pobre apenas alcanza el 1.5\%".

Asimismo, la regresividad queda igualmente patente en los resultados. La política de subsidios al consumo (eléctrico residencial, gasolinas y las exenciones al IVA) invariablemente beneficia a la población con mayores ingresos, pues solo el $34 \%$ del gasto destinado a combatir la desigualdad es progresivo (Coneval, 2014). Otro tanto ocurre con la política de salario mínimo. Como arguyen Bosch y Manacorda (2010) lejos de ser un mecanismo para reducir el "gap" en las rentas del trabajo consigue incrementar la desigualdad. Hecho que se puede explicar a partir del exponencial incremento salarial de las rentas más altas, así como del alto grado de informalidad en la que se generan las rentas de los grupos de menores ingresos.

\section{Conclusiones}

El rol de la desigualdad en la nueva agenda mundial del desarrollo sostenible parece incuestionable. En un primer apartado se ha analizado cómo las implicaciones de la desigualdad en el diseño de los nuevos objetivos sostenibles suponen no solo un nuevo impulso a la consecución de un mundo libre de pobreza en todas sus dimensiones, sino que con igual energía avanza hacia la plena “operacionalización” del desarrollo como libertad real.

Asimismo, gracias al concepto de resiliencia humana la nueva agenda consigue superar los límites presentes en el concepto de libertad seniana de oportunidad para expandirse a una dimensión de procesos, e incorporar aquellos fenómenos que determinan el funcionamiento real de los individuos. De todos aquellos "estados sociales" que lo condicionan, se ha tomado como variable focal de esta investigación la desigualdad. Las razones derivan de sus extensas implicaciones teóricas y prácticas para la nueva agenda y la región de la frontera norte mexicana como caso particular de estudio. En un segundo apartado se realizó un ejercicio de prospectiva por medio de una serie de indicadores del milenio con el fin de conocer el "estado de la

Manuel Antonio Jiménez Castillo

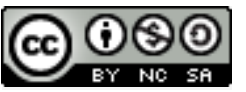

Revista Economía y Sociedad by Universidad Nacional is licensed under a CreativeCommons Reconocimiento-NoComercial- 
cuestión" del desarrollo humano en la FNM. Al ser conscientes de que logro (indicadores ODM) y sostenibilidad en el logro (indicadores ODS) no tiene el mismo alcance, la ausencia de datos estadísticos para estos últimos exigió dibujar un mapa inconsistente, aunque no por ello inútil para los propósitos de esta investigación.

A través de la metodología de las "tres erres" se reveló como la FNM atesora problemas estructurales "de logros" en las cuestiones específicas de género (ODS 5) y empleabilidad (ODS 8). En las referidas a saneamiento (ODS 7) y mortalidad infantil (ODS 3 ) se requiere continuar en la senda de avances ya cosechados que si bien son positivos no garantiza por sí solos su sostenibilidad futura.

Si en la cuestión de los logros, a la FNM se le atribuyen notables lagunas por superar, hablar de sostenibilidad en el logro implica no solo suspender su éxito a un "Big-Push" como refiere Jeffry Sachs, sino al hecho de examinar la efectividad de aquellos instrumentos dados para tal fin. Precisamente, el extraordinario nivel de desigualdad en la FNM apunta a una insuficiencia estructural sobre las posibilidades para su cumplimiento.

A través del concepto de "asimetría de poder" se examinó especulativamente cómo la desigualdad en esta región del mundo lejos de expresarse como resultado actúa como "espiral viciosa", puesto que condiciona la naturaleza de la política económica y social. A partir de esta tesis se dedujo de qué modo la naturaleza de las políticas dada a su combatimiento se encuentra particularmente condicionada por la desigualdad haciendo que aquellas, lejos de minimizar su impacto, se conviertan en instrumentos de sostenibilidad de la propia desigualdad. Por todo ello, una de las barreras más sobresaliente que la FNM encuentra para dar cumplimiento a tales objetivos no vendría hipotecado a un acto "adecuado" de implementación de las políticas de desarrollo, sino más bien a la posibilidad fáctica para poder revertir tales indeseables consecuencias asociadas al grado de desigualdad en el que quedan inmersas.

\section{Referencias}

Bosch, M., \& Manacorda, M. (2010). Minimum Wages and Earnings Inequality in Urban Mexico. American Economic Journal, 2(4), 128-49. http://dx.doi.org/10.1257/app.2.4.128

Cejudo, R. (2007). Capacidades y libertad. Una aproximación a la teoría de Amartya Sen. Revista Internacional de Sociología, 65(47), 9-22. Recuperado de http://revintsociologia.revistas.csic.es/index.php/revintsociologia/article/viewArticle/50

Comisión Económica para América Latina y el Caribe (2010). Panorama social de América Latina(Informe anual). Recuperado de http://www.cepal.org/es/publicaciones/1236-panorama-social-deamerica-latina-2010

14

Manuel Antonio Jiménez Castillo

(c) (P) 8 (2)

Revista Economía y Sociedad by universidad Nacionalislicensedunder a CreativeCommons Reconocimiento-NoComercial- 
Consejo nacional de evaluación de la política social. (2014). Pobreza en México.Recuperado de http://www.coneval.org.mx/Medicion/MP/Paginas/Pobreza 2014.aspx

Cortés, F., \& De Oliveria, O. (2010). Los grandes problemas de México Desigualdad Social.Recuperado de http://2010.colmex.mx/16tomos/V.pdf

Deneulin, S. (2006). Individual Well-being, Migration Remittances and the Common Good. European Journal of Development Research, 18(1), 45-61. doi: http://dx.doi.org/10.1080/09578810600572353

Deneulin, S. (2011). Development and the limits of Amartya Sen's The Idea of Justice. Third World Quarterly, 32(4), 787-797.doi: http://dx.doi.org/10.1080/01436597.2011.567008

Dungey, M., \& Jacobs, J. (2010). Financial crisis in Asia: concordance by asset market or country? (Discussion Paper 12). Recuperado del sitio de internet de School of Economics and Finance: http://eprints.utas.edu.au/10575/1/DP2010 12 Dungey Jacobs Lestano Receipts October 20 10.pdf

Esquivel, G. (2010). The dynamics of income inequality in Mexico since NAFTA(Serie de documentos de trabajo IX-2010). Recuperado de http://cee.colmex.mx/documentos/documentos-detrabajo/2010/dt20109.pdf

Fukuda-Parr, S. (2010). Reducing Inequality-The Missing MDG: A content Review of PRSPs and Bilateral Donor Policy Statements. IDS Bulletin, 41(1), 26-33. doi: http://dx.doi.org/10.1111/j.17595436.2010.00100.x

Fukuda-Parr, S. (2012). Recapturing the Narrative of International Development(Research Paper No. 2012-5).Recuperado del sitio de internet United Nations Research Institute for Social Development:

http://www.unrisd.org/80256B3C005BCCF9/(httpAuxPages)/026AC192E40B70CEC1257A370032 E678/\$file/Fukuda-Parr.pdf

Fukuda-Parr, S., Ely , A., \& Greenstein, J. (2014). The Power of Numbers: A Critical Review of Millenium Development Goal Targets for Human Development and Human Rights. Journal of Human Development and Capabilities, 15(2-3), 1-13. doi: http://dx.doi.org/10.1080/19452829.2013.864622

Jiménez Castillo, M. (2016). Amartya Sen frente al espejo social de la libertad. Revista internacional de sociología, 73(3). doi: http://dx.doi.org/10.3989/ris.2016.74.3.038 
Johnson, S., Kaufmann, D., \& Zoido, P. (1998). Regulatory discretion and the unofficial economy. American Economic Review, 82(2), 387-392. http://papers.ssrn.com/sol3/papers.cfm?abstract id=1116727

Le Blanc, D. (2015). Towards integration at last? The sustainable development goals as a network of targets (DESA Working Paper No. 141). Recuperado de sitio de internet Departament of Economic \& Social Affairs: http://www.un.org/esa/desa/papers/2015/wp141 2015.pdf

Martínez, I. (2013). La cooperación descentralizada a debate. Barcelona: CIDOB. Recuperado de http://www.cidob.org/es/publicaciones/serie de publicacion/monografias/monografias/la coo peracion descentralizada a debate la eficacia de la ayuda $y$ el post 2015

Max-Neef, M. (1986). Desarrollo a escala humana conceptos, aplicaciones y algunas reflexiones. Recuperado de http://www.max-neef.cl/descargas/Max Neef-Desarrollo a escala humana.pdf

Nayyar, D. (2011). The MDGs Beyond 2015 (Research Paper, 38). Recuperado del sitio de internet de South Centre: http://www.southcentre.int/wp-content/uploads/2013/05/RP38 MDGsbeyond2015 EN.pdf

Nussbaum, M. (2012). Crear capacidades: Propuesta para el desarrollo humano.Recuperado de https://books.google.es/books/about/Crear capacidades.html?hl=es\&id=RWOKtgAACAAJ

Panel of Eminent Person (2013). A new Global Partnership: Erradicate Poverty and Transform Economies Through Sustainable Development de https://sustainabledevelopment.un.org/index.php?page=view \&type $=400 \& n r=893 \&$ menu $=1561$

Programa Naciones Unidas para el Desarrollo (1990). Human Development Report.Recuperado de http://hdr.undp.org/sites/default/files/reports/219/hdr 1990 en complete nostats.pdf

Programa Naciones Unidas para el Desarrollo. nud. (1999). La mundialización con rostro humano. Recuperado de http://hdr.undp.org/es/content/informe-sobre-desarrollo-humano-1999

Programa Naciones Unidas para el Desarrollo (2014). Sostener el progreso humano: reducir vulnerabilidades $y \quad$ construir resilencias. Recuperado de http://www.undp.org/content/dam/undp/library/corporate/HDR/2014HDR/HDR-2014Spanish.pdf

Rosignolo, D., \& Gómez, J. (2011). Impacto de las políticas tributarias sobre la equidad en países de América Latina. En J. Nun (comp.) La desigualdad y los impuestos. Introducción para no especialistas. Recuperado de http://www.peronlibros.com.ar/content/nun-jos\%C3\%A9-ladesigualdad-y-los-impuestos-introducci\%C3\%B3n-para-no-especialistas

16

Manuel Antonio Jiménez Castillo

(c) (i) $\$$ (O)

Revista Economía y Sociedad by universidad Nacionalislicensedunder a CreativeCommons Reconocimiento-NoComercial- 
Scott, J. (2010). Subsidios agrícolas en México, ¿quién gana y cuánto? En J. Fox, \& H. Libby (comp.), Subsidios para la desigualdad. Las políticas públicas del maíz en México. Recuperado dehttps://www.wilsoncenter.org/sites/default/files/Subsidios\%20Para\%20La\%20Desigualdad.pd $\underline{f}$

Scott, J. (2011). Gasto público para la equidad: del estado truncado hacia el estado de bienestar universal. México: Working Paper for México Evalúa. http://frentealapobreza.org.mx/wpcontent/uploads/2015/04/10-puntos.pdf

Sen,

A.

(1999).

Development

as

Freedom.

Recuperado

dehttps://books.google.co.cr/books/about/Development as Freedom.html?id=NQs75PEa618C \&redir esc=y

Sen,

A.

(2009).

The

Idea

of

Justice. Recuperado

de

https://books.google.co.cr/books/about/The Idea of Justice.html?id=engMd ze6RMC\&redir e $\underline{\mathrm{sC}=\mathrm{y}}$

Vandemoortele, J. (2014). Post-2015 agenda: mission impossible? Development Studies Research, 1(1), 223-232. doi: http://dx.doi.org/10.1080/21665095.2014.943415 